\title{
Sistema de extraccion y tratamiento de metano
}

\section{Methane extraction and treatment system}

CANTÚ-MUNGUÍA Irma Adriana, GARAY-DE LA O, Albino, HERNÁNDEZ-CERVANTES, Aldo Aarón y GARCIABADA-SILVA, Gabriel

\author{
Instituto Tecnológico de Jalisco José Mario Molina Pasquel y Enriquez \\ ID 1 ${ }^{\text {er }}$ Autor: Irma Adriana, Cantú-Munguía / ORC ID: 0000-0002-1904-421X \\ ID $1^{\text {er }}$ Coautor: Albino, Garay-De La O / ORC ID: 0000-0002-3321-7594 \\ ID $2^{\text {do }}$ Coautor: Aldo Aarón, Hernández-Cervantes / ORC ID: 0000-0001-8060-5509 \\ ID $3^{\text {er }}$ Coautor: Gabriel, Garciabada-Silva / ORC ID: 0000-0002-7083-871X
}

DOI: $10.35429 / J R E .2019 .10 .3 .7 .18$

Recibido 3 de Julio, 2019; Aceptado Septiembre 30, 2019

\begin{abstract}
Resumen
Actualmente los rellenos sanitarios son considerados fuentes emisores de contaminantes atmosféricas. El biogás, producto de la descomposición biológica de los residuos orgánicos, incorpora en su mayoría metano (CH4) y dióxido de carbono $(\mathrm{CO} 2)$, que son gases de efecto invernadero. Un gran número de rellenos sanitarios municipales no poseen un control sobre las emisiones de gas metano a la superficie. Este proyecto tiene como fundamento realizar la construcción de un sistema prototipo portátil de extracción y tratamiento del gas metano proveniente de los rellenos sanitarios. Como objetivo se estableció el fabricar y construir un sistema prototipo portátil de extracción y tratamiento del gas metano proveniente de los rellenos sanitarios municipales. Que implemente las secciones y dispositivos necesarios para tratar el gas y de esta manera facilitar su posterior uso como combustible en sistemas de calefacción, uso doméstico y/o maquinas térmicas de combustión interna y externa. Utilizando para este fin un sistema de extracción de gases, separación de sólidos, proceso de des humidificación y desulfuración. En el sistema las variables como el porcentaje de metano, temperatura y humedad son monitoreadas, con el fin de darle una estabilidad al proceso de tratamiento del gas.
\end{abstract}

Biogás, Metano, Aprovechamiento

\begin{abstract}
Currently sanitary landfills are considered sources of atmospheric pollutants. Biogas, a product of the biological decomposition of organic waste, incorporates mostly methane $(\mathrm{CH} 4)$ and carbon dioxide $(\mathrm{CO} 2)$, which are greenhouse gases. A large number of municipal sanitary landfills do not have control over methane gas emissions to the surface. This project is based on the construction of a portable prototype system for the extraction and treatment of methane gas from landfills. The objective was to manufacture and build a portable prototype system for the extraction and treatment of methane gas from municipal sanitary landfills. That it implements the necessary sections and devices to treat the gas and in this way facilitate its later use as fuel in heating systems, domestic use and / or internal and external combustion thermal machines. Using for this purpose a system of gas extraction, separation of solids, de-humidification and desulphurisation process. In the system variables such as the percentage of methane, temperature and humidity are monitored, in order to give a stability to the gas treatment process.
\end{abstract}

Biogas, Methane, Utilization

Citación: CANTÚ-MUNGUÍA Irma Adriana, GARAY-DE LA O, Albino, HERNÁNDEZ-CERVANTES, Aldo Aarón y GARCIABADA-SILVA, Gabriel. Sistema de extraccion y tratamiento de metano. Revista de Energías Renovables. 2019 3-10: 718

\footnotetext{
*Correspondencia al Autor (Correo electrónico: irma.cantu@ vallarta.tecmm.edu.mx)

$\dagger$ Investigador contribuyendo como primer autor.
} 


\section{Introducción}

Actualmente los rellenos sanitarios son considerados fuentes emisores de contaminantes atmosféricas. El biogás, producto de la descomposición biológica de los residuos orgánicos, incorpora en su mayoría metano $\left(\mathrm{CH}_{4}\right)$ y dióxido de carbono $\left(\mathrm{CO}_{2}\right)$, que son gases de efecto invernadero. Un gran número de rellenos sanitarios municipales no poseen un control sobre las emisiones de gas metano a la superficie.

El gas metano es uno de los principales causantes del efecto invernadero a nivel mundial, y con la contribución de los rellenos sanitarios de liberar constantemente gas metano a la atmosfera sin ningún control o aprovechamiento, este problema se incrementa de manera alarmante.

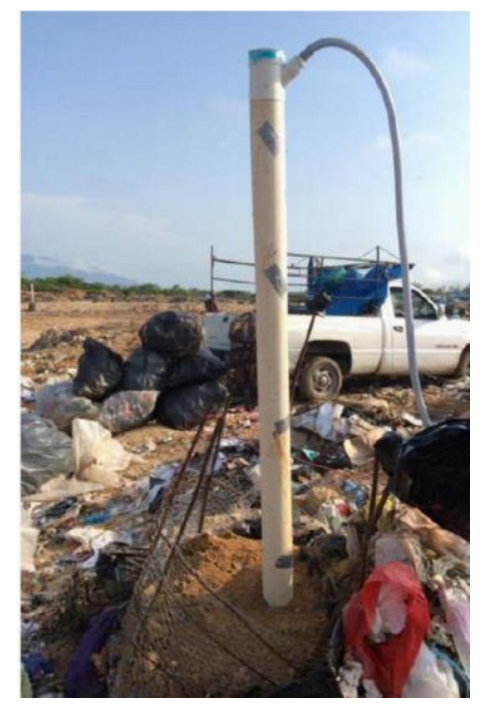

Figura 1 Salida de Biogás en relleno sanitario. Fuente: Elaboración Propia

Por lo que el metano, siendo un gas de efecto invernadero, posee un potencial de calentamiento; que es una medida de la eficiencia de un gas para retener calor en la atmósfera.

En Puerto Vallarta Jalisco, municipio donde se encuentra el relleno sanitario "El Gavilán" no posee un control sobre parte de las emisiones de gas metano a la superficie. Esto trae como resultado la necesidad de proporcionar un medio por el cual el gas metano sea aprovechado y almacenado para su posterior uso.
Cabe destacar que vertedero municipal de Puerto Vallarta requiere de un sistema que evite que el gas metano permanezca emitiéndose de forma incontrolada, integrándose a la atmosfera como contaminante y desperdiciándose su uso energético. Cuando el biogás tiene un contenido de metano superior al $45 \%$, este es inflamable y puede ser utilizado como fuente de energía. Por lo cual, el gas metano adquiere importancia energética debido a su alta inflamabilidad y amplia variedad de usos como biocombustible. Por ejemplo; calefacción, uso doméstico o máquinas térmicas de combustión interna y externa para generación de energía mecánica y/o eléctrica. (Mechile, Pnud, Fao, \& Gef, 2011).

El objetivo de este proyecto es fabricar y construir un sistema prototipo portátil de extracción y tratamiento del gas metano proveniente de los rellenos sanitarios municipales. Que implemente las secciones y dispositivos necesarios para tratar el gas y de esta manera facilitar su posterior uso como combustible en sistemas de calefacción, uso doméstico y/o maquinas térmicas de combustión interna y externa. El metano (CH4) conformado por cuatro átomos de hidrógeno unidos al átomo de carbono central, pertenece a la familia de los alcanos; hidrocarburos que solo contienen enlaces sencillos. Posee un punto de ebullición de $-164^{\circ} \mathrm{C}$, punto de fusión de $-183^{\circ} \mathrm{C}$ y una densidad de $0.55 \mathrm{~g} / \mathrm{ml}$ a la temperatura de su punto de ebullición. Por lo tanto, a temperatura ambiente y presión atmosférica se presenta en el estado gaseoso. Es incoloro, inodoro, altamente inflamable y no tóxico, es uno de los compuestos orgánicos más sencillos que existen. (Wade, 2012)

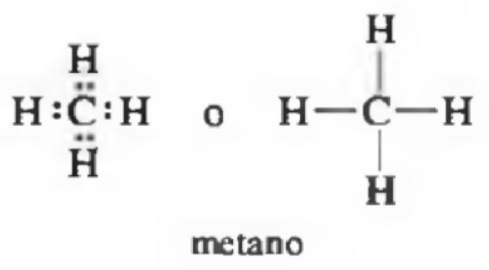

Figura 2 Metano representado en estructuras de Lewis (Wade, 2012)

La gran mayoría del metano en la Tierra es producido, directa o indirectamente, por microorganismos. La metanogénesis, es decir la producción biológica de metano, es un proceso característico de las denominadas arqueas metanógenas (anaerobias estrictas). (Garzón Pinto, 2017) 
Estos microorganismos son muy abundantes en sedimentos marinos, pantanos, tracto digestivo de animales rumiantes, digestores de aguas residuales e instalaciones artificiales de biodegradación; estas últimas incluyen biodigestores y vertederos, los cuales poseen ambientes anaerobios en su interior donde gracias a la acción metabólica de estos microorganismos la materia orgánica que contienen entra en descomposición produciéndose el metano. (Garzón Pinto, 2017)

La combustión es la reacción más común de los alcanos; el metano, proveniente de esta familia, es altamente inflamable. Debido a esta propiedad es que posee un prominente interés en su uso como fuente de energía calorífica.

\section{El biogás}

El gas producido en vertederos no es en su totalidad gas metano, sino una mezcla de diferentes gases, por eso es llamado de forma general como "biogás". No debe confundirse con el gas natural, porque el gas natural es un producto proveniente del tratamiento del petróleo, aunque está compuesto en un $70 \%$ por metano, posee aproximadamente un $10 \%$ de etano y $15 \%$ de propano; gases provenientes de los yacimientos de petróleo o carbón. (Wade, 2012)

El biogás está constituido principalmente por los gases dióxido de carbono (CO2), metano (CH4), vapor de agua (H2O), otras pequeñas cantidades de gases como Hidrógeno $(\mathrm{H})$, Nitrógeno (N) y Sulfuro de hidrógeno (H2S), y trazas de compuestos orgánicos volátiles (COV). (Ortega, Rodríguez, Fernández, \& Bárcenas, 2015).

\section{Antecedentes}

El 10 de octubre del 2003 se publicó en el Diario Oficial de la Federación, con carácter de proyecto la Norma Oficial Mexicana PROYNOM-083-SEMARNAT2003. Después, habiéndose cumplido con el procedimiento establecido en la Ley Federal sobre Metrología y Normalización el Comité Consultivo Nacional de Normalización de Medio Ambiente y Recursos Naturales, en sesión ordinaria de fecha 9 de junio de 2004, aprobó la Norma Oficial Mexicana NOM-083-SEMARNAT-2003, denominada.
Especificaciones de protección ambiental para la selección del sitio, diseño, construcción, operación, monitoreo, clausura y obras complementarias de un sitio de disposición final de residuos sólidos urbanos y de manejo especial. (NORMA, 2004).

La presente Norma Oficial Mexicana de acuerdo a su objetivo planteado; establece las especificaciones de selección del sitio, el diseño, construcción, operación, monitoreo, clausura y obras complementarias de un sitio de disposición final de residuos sólidos urbanos y de manejo especial. De acuerdo a la problemática causada por los gases resultantes de los procesos biológicos de los rellenos sanitarios, esta norma como lo vimos anteriormente en el apartado de justificación, en la sección 7.2 indica el tomar las medidas necesarias para la extracción, captación, conducción y control del biogás.

Hasta el año 2016 en un registro hecho por la Secretaría de Energía, SENER, de acuerdo a la revista digital Capital México (Pichardo, 2016), cumpliendo el requisito que especifica la NOM-083-SEMARNAT-2003 existen 16 centrales de captación y aprovechamiento del gas metano distribuidas en 11 estados: Coahuila, Chihuahua, Veracruz, Aguascalientes, Nuevo León, Jalisco, Durango, Estado de México, San Luis Potosí, Tabasco y Morelos.

\section{Medición del gas metano}

Al ser capturado el gas metano, este debe ser cuantificado en sus concentraciones para obtener un mayor aprovechamiento de sus propiedades como combustible. Existen sensores los cuales actúan como medidores de estas concentraciones otorgando al sistema de captación un mayor control en el proceso.

Los dispositivos electrónicos para medición de la concentración de metano hacen uso de diversas técnicas. Estas incluyen variaciones en semiconductores de óxidos metálicos (MOS) como los sensores, empleo de polímeros y nanotubos de carbono, sustratos con propiedades de absorción específicas a ciertas frecuencias (RF), espectroscopia en las bandas del infrarrojo, calorimetría por pellistores, cambios acústicos y extendidamente, la cromatografía. (Vaquerano Pineda, Salazar Rojas, \& Porras Acosta, 2016).El tipo de medición que se realizó en la presente investigación es utilizando los semiconductores de óxidos metálicos (MOS).

CANTÚ-MUNGUÍA Irma Adriana, GARAY-DE LA O, Albino, HERNÁNDEZ-CERVANTES, Aldo Aarón y GARCIABADASILVA, Gabriel. Sistema de extraccion y tratamiento de metano. Revista de Energías Renovables. 2019 


\section{Sensores a base de semiconductores de óxidos metálicos}

Estos sensores reaccionan ante la presencia de gases en el ambiente reduciendo su resistencia eléctrica mediante un material semiconductor a base de óxido de estaño $\left(\mathrm{SnO}_{2}\right)$, el cual al entrar en contacto con algún gas combustible modifica sus propiedades conductivas, es decir la resistencia sensitiva se reduce, por consiguiente, aumenta el voltaje de respuesta.

De acuerdo a (Ordoñez Mendoza, Fitz Rodríguez, \& Espinosa Solares, 2017); el óxido de estaño es un material capaz de modificar su conductividad ya que interactúa con las moléculas de gas presentes en el ambiente. Al absorber el oxígeno, la banda de conducción del material expulsa los electrones absorbidos en forma de iones, lo cual deja una mayor superficie para la absorción de más electrones provenientes de los gases. La sensibilidad de los materiales basados en óxidos metálicos cambia de acuerdo a los factores que influyen en las reacciones de su superficie, tales como composición química, modificación de la superficie, arreglos micro estructural, temperatura y humedad en el medio de interacción.

Dentro del mercado podemos encontrar modelos de sensores económicos del tipo MOS, de entre estos destaca la gama de sensores MQ. Todos los modelos MQ son analógicos ya que su funcionamiento se basa en un divisor de voltaje. Poseen un calentador para elevar la temperatura de su material sensitivo y este pueda medir de forma más exacta las concentraciones del gas de interés.

La tensión a la que estos sensores funcionan es por lo regular a $5 \mathrm{v} \mathrm{CD}$, su consumo puede ser elevado dentro del rango promedio que proveen los sistemas embebidos, llegando a valores de $800 \mathrm{~mW}$ por lo que es necesario proporcionar una fuente de alimentación externa de mayor capacidad. (Llamas, 2016). No obstante, es necesario consultar el Datasheet de cada sensor MQ particular antes de emplearlo para detallar sus especificaciones técnicas, especialmente la tensión de alimentación del calentador, consumo, el tiempo de calentamiento y la curva de sensibilidad del sensor a ciertos gases. Los sensores MQ-2 y MQ-4 regularmente son usados para la medición del gas metano.
Estos sensores trabajan a $5 \mathrm{v}$, con una resistencia de calentamiento a $5 \mathrm{v}$, bajo condiciones ambientales de hasta 10000 ppm (MQ-4) y 20000 ppm (MQ-2) de metano, en condiciones estándares de $21 \%$ de oxígeno, a una temperatura de $20^{\circ} \mathrm{C}$ y $65 \%$ de humedad relativa. (Ordoñez Mendoza, Fitz Rodríguez, \& Espinosa Solares, 2017)

\section{Sensor MQ-4}

De acuerdo a (Rodríguez, Delgado, Aroca Trujillo, \& Navarrete Ramos, 2018); Este es un sensor para detectar gas metano principalmente. El MQ-4 puede detectar concentraciones de 300 a 10000 ppm. Este transductor contiene una alta sensibilidad y un tiempo de respuesta rápido. El resultado tiene una señal analógica que es el signo que nos permite analizar el comportamiento. El circuito de interfaz es muy simple, todo tiene que ver con la fuente de alimentación de $5 \mathrm{~V}$, agrega una resistencia de carga y conecta la salida al convertidor analógico-digital. Conforme a (Rodríguez, Delgado, Aroca Trujillo, \& Navarrete Ramos, 2018); El valor de resistencia del MQ-4 es diferente de varias concentraciones de gas. Por lo tanto, el ajuste de sensibilidad es muy necesario. Se recomienda calibrar el detector para 5000 ppm de concentración de $\mathrm{CH}_{4}$ en aire y usar el valor de resistencia de carga $(\mathrm{RL}) \mathrm{de}$ aproximadamente $20 \mathrm{~K} \Omega(10 \mathrm{~K} \Omega$ a $47 \mathrm{~K} \Omega)$.

\section{Inversor de modulación de ancho de pulso}

Para realizar las pruebas necesarias al prototipo debe contarse con una fuente de alimentación independiente de $127 \mathrm{v}$ CA que provea energía eléctrica a los extractores y sistema de medición de variables. El sistema de extracción de metano está diseñado para operar en vertederos, áreas dónde no es sencillo contar con alguna fuente de alimentación de energía eléctrica. Tal problema puede ser resuelto implementando al prototipo un inversor de modulación de ancho de pulso, que es un convertidor de $\mathrm{CD}$ a $\mathrm{CA}$ alimentado por baterías.

De acuerdo a (Muhammad H, 1993); La función de un inversor es cambiar un voltaje de entrada en CD a un voltaje simétrico de salida en CA, con la magnitud y frecuencia deseadas. Tanto el voltaje de salida como la frecuencia pueden ser fijos o variables. Para realizar la variación, se controla la modulación del ancho de pulso (PWM). 
Para que un inversor pueda ser considerado ideal la forma de onda del voltaje de salida debe ser senoidal, sin embargo, en la realidad no se llega a esta forma de manera perfecta. Para aplicaciones de mediana y baja potencia, se pueden aceptar los voltajes de onda cuadrada o casi cuadrada; para aplicaciones de alta potencia, son necesarias las formas de onda senoidales de baja distorsión. (Muhammad H, 1993)

Consultando a (Muhammad H, 1993) la clasificación de los inversores se distingue en dos tipos: Inversores monofásicos e inversores trifásicos. Dependiendo la aplicación que se le vaya a dar los inversores deben constituirse por dispositivos electrónicos de potencia con activación y desactivación controlada, es decir BJT, MOSFET, IGBT, MCT, SIT, GTO o tiristores de conmutación forzada. Para poder realizar la variación del voltaje o frecuencia se implementa un dispositivo que genere señales de control PWM, puede ser un generador de pulsos u oscilador. Una vez formada la onda cuadrada o senoidal de baja distorsión esta pasa por un transformador el cual eleva el voltaje y al mismo tiempo suaviza la onda. Otorgando la tensión alterna deseada en las terminales del devanado opuesto.

\section{Desarrollo}

\section{Primera propuesta para el diseño del prototipo}

Este diseño en 2D fue presentado en el anteproyecto, siendo la primera propuesta del prototipo. Durante el desarrollo del proyecto este fue tomando varios cambios en los dispositivos a utilizar como en su orden, más el fin siguió siendo el mismo; extraer gas metano del biogás proveniente de los basureros y realizar su tratamiento.

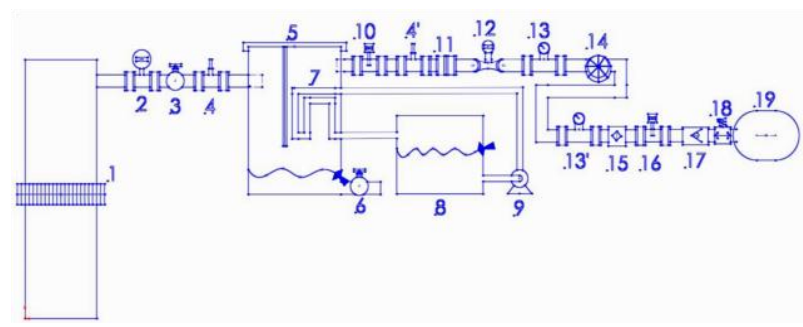

Figura 2 Primera propuesta 2D

Fuente: Elaboración Propia

\section{Elementos de la primera propuesta}

$1=$ Malla para salida de metano del vertedero

$2=$ Sensor de $\%$ Metano

$3=$ Válvula de bola

4y 4' = Sensores de temperatura de entrada y salida (DS18B20)

$5=$ Cárcamo de condensado.

6= Válvula de desagüe del cárcamo

$7=$ Condensador tipo de doble tubo

$8=$ Depósito de refrigerante

$9=$ Bomba de refrigerante

$10=$ Sensor de humedad

11= Acople PVC-cobre

$12=$ Caudalímetro

13y 13' = Manómetro de baja y alta (0-30PSI)

$14=$ Compresor rotativo de paletas (Para gases combustibles)

15= Filtro de carbón activado para absorción de $\mathrm{CO} 2$ y desulfuración.

16= Sensor de sulfuro de hidrógeno y dióxido de carbono

$17=$ Válvula anti retorno

$18=$ Válvula reguladora de presión con escape

$19=$ Tanque de almacenamiento

\section{Estimación del funcionamiento del primer diseño}

Tomando como referencia al documento "Modelo para el diseño de sistemas de captación y aprovechamiento de biogás producido en rellenos sanitarios" de la Facultad de ciencias físicas y matemáticas, departamento de Ingeniería Mecánica de la Universidad de Chile. (López Arriaza \& Nuñez Lazo, 2016)

Se determina que un sistema de extracción y tratamiento de gas metano, consiste en varios dispositivos eléctricos, mecánicos, electrónicos y neumáticos conectados entre sí para lograr la captación y aprovechamiento del gas metano, evitando así su integración a la atmosfera. De acuerdo a normas de construcción, cada vertedero debe contar con salidas de gas metano aproximadamente cada 50 metros cuadrados, para evitar impedir la salida de los gases y estos provoquen grandes accidentes en una salida brusca por liberar presión. El gas es extraído directamente de las salidas en vertederos. (NOM 083, 2004). 
La extracción del gas se realiza gracias a la caída de presión (vacío) que genera el compresor. El sistema comienza con una malla trampa de sólidos, a su salida este tendrá un medidor de porcentaje de metano. Para que el gas pueda ser considerado eficiente debe contener aproximadamente un $60 \%$ de metano (CH4) y un $40 \%$ de dióxido de carbono (CO2). $\mathrm{Si}$ el metano baja de ese porcentaje, el nivel de energía calorífica que puede generar es ineficiente.

En la siguiente sección la tubería es reducida de su diámetro, después está tendrá un aumento elevado de su diámetro al entrar al cárcamo de condensado, expandiendo bruscamente el gas haciendo el efecto similar un sistema de refrigeración, el gas expandido bruscamente debe disminuir su temperatura y entrar a un condensador, enfriado por agua, que debe disminuir más la temperatura hasta llegar al punto de rocío. La sección del condensador separará el vapor de agua del metano, disminuyendo en gran manera su nivel de humedad. A la salida del condensador tenemos un medidor de porcentaje de humedad y un sensor de temperatura.

El vapor de agua que procede de la extracción del metano, con el tiempo se vuelve un corrosivo muy dañino para las tuberías y partes móviles del sistema, además que su unión con el H2S resulta una reacción química que da como resultado $\mathrm{H} 2 \mathrm{SO} 4$, un ácido altamente corrosivo. El objetivo es tener, a la salida del condensador, el nivel más mínimo de humedad que sea posible.

En la siguiente fase tenemos un compresor rotativo de paletas para gases combustibles, que es la potencia del sistema, creando un vacío para extraer el gas y un incremento de presión para su almacenamiento. Cabe destacar que el almacenamiento no es objeto de estudio en el presente proyecto, proponiendo solo un diseño para futuras implementaciones de este proceso.

Ya en la zona de alta presión, el gas pasa por un filtro de carbón activado, el cual cumple la función de la absorción del dióxido de carbono y la desulfuración el gas. (Ortega, Rodríguez, Fernández, \& Bárcenas, 2015)
A la salida del filtro contamos con un medidor de porcentaje de Sulfuro de hidrógeno, una válvula anti retorno, una válvula de alivio y finalmente el depósito de almacenamiento. La válvula anti retorno impide que el gas salga del depósito y la válvula de alivio actúa como un sistema de seguridad ante presiones elevadas.

De entre los procesos necesarios para el tratamiento del gas metano destacan el proceso de condensación del vapor de agua y la desulfuración. Siendo estos profundamente importantes para incrementar la calidad del biocombustible y excluir los efectos corrosivos que el agua y el $\mathrm{H} 2 \mathrm{~S}$ puedan ocasionar a las máquinas y dispositivos que lo utilicen. (Ortega, Rodríguez, Fernández.

El biogás, después de haberse producido en las zonas anaerobias del vertedero, de acuerdo a las propiedades físicas de sus gases constituyentes en ocupar todo el espacio disponible y ser menos densos que el aire, el biogás se eleva buscando medios de escape. Para esto, en la construcción de cada vertedero están contemplados pozos cada cierta área de metros cuadrados, por donde el biogás tiene su circulación en tuberías. Estos tubos de PVC sanitario, regularmente de $4 \frac{1}{2}$ " de diámetro, son perforados y empotrados en los pozos, recubiertos de grava y arena liviana para evitar el contacto con los lixiviados o ser aplastados por los mismos desechos. (López Arriaza \& Nuñez Lazo, 2016) . Justo en el terminante de los tubos, a la superficie, es dónde comienza la extracción y tratamiento del gas metano por medio del prototipo propuesto.

\section{Metodología}

La primera sección la constituyen la trampa de sólidos y los extractores. La función de la trampa de solidos es impedir que las trazas de compuestos orgánicos volátiles entren al sistema. Para este propósito en el prototipo construido se empleó una malla de mosquitero fijada a un cople PVC 41/2" a 41/2", con orificios suficientemente pequeños para impedir que los sólidos volátiles entre al sistema. Para su mantenimiento este debe ser inspeccionado y limpiado cada cierto tiempo, dependiendo el período de uso del sistema. 


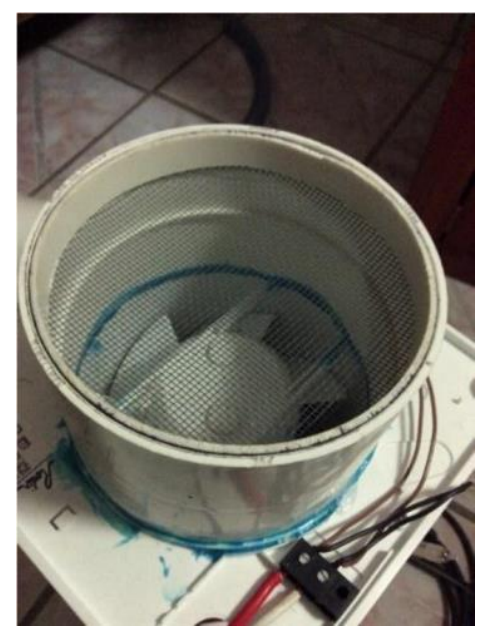

Figura 3 Trampa de sólidos Fuente: Elaboración Propia

Para la implementación de los extractores se tuvo que buscar los que tuvieran la medida preferentemente igual al cople de $4 \frac{1}{2}$ ". Estos se consiguieron en un depósito de material electrónico y en buenas condiciones. El prototipo físico en su primera fase de construcción comprendió tres extractores, después fue añadido uno más, colocados y fijados en serie con pegamento para PVC.

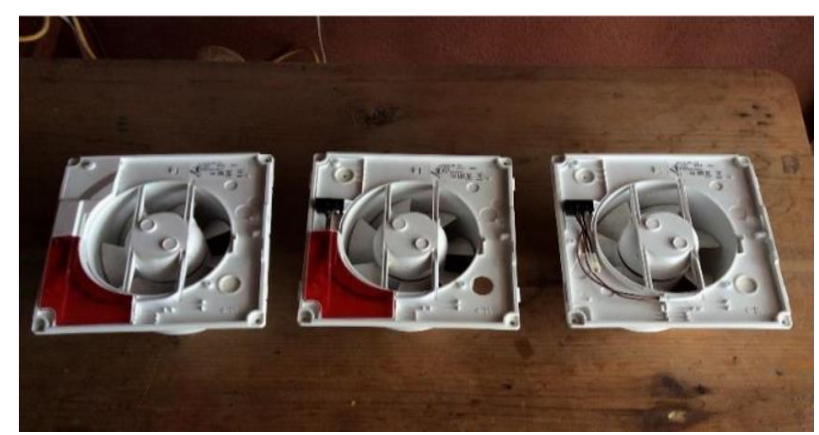

Figura 4 Extractores

Fuente: Elaboración Propia

Para el seguimiento de la conducción del biogás este debe pasar a una sección transversal más angosta, tanto por el proceso físico que esto genera en el sistema como la ineficaz utilización de elementos que se acoplen a un diámetro tan grande como el de 4 1/2”. Por lo cual se empleó una reducción en diagonal de 4" a 1", posteriormente dos reducciones de 1 " a $3 / 4 "$ unidas a una manguera de $3 / 4$ " y $50 \mathrm{~cm} \mathrm{de}$ longitud.

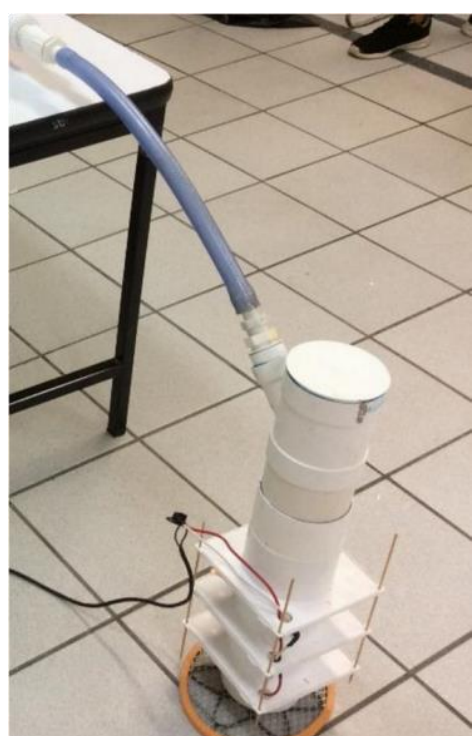

Figura 5 Sección de trampa de sólidos y extractores Fuente: Elaboración Propia

\section{Construcción de la sección de medición de temperatura y humedad}

La siguiente sección en ser integrada al prototipo físico fue la que realiza la función de medir las variables de temperatura y humedad del biogás. Fue seleccionada tubería PVC hidráulico de 1 $1 / 2$ " y el sensor digital DHT11 para medición de la humedad y temperatura.

El elemento diseñado para ser implementado en el sistema y contener al sensor DHT11, fue una abrazadera conformada por dos piezas las cuales se unen físicamente por medio de tornillos con su respectiva tuerca. Poseen desde una perspectiva radial una abertura central donde es acoplada la tubería de 1 1/2" y de manera axial en el centro, una de ellas posee un espaciamiento en forma de prisma cuadrangular donde es colocado el sensor, con una guía por la que pueden ser guiados los cables que alimentan y comunican al sensor con la tarjeta.

Se procedió a realizar el programa para el monitoreo del sensor. El cual se presenta a continuación. 


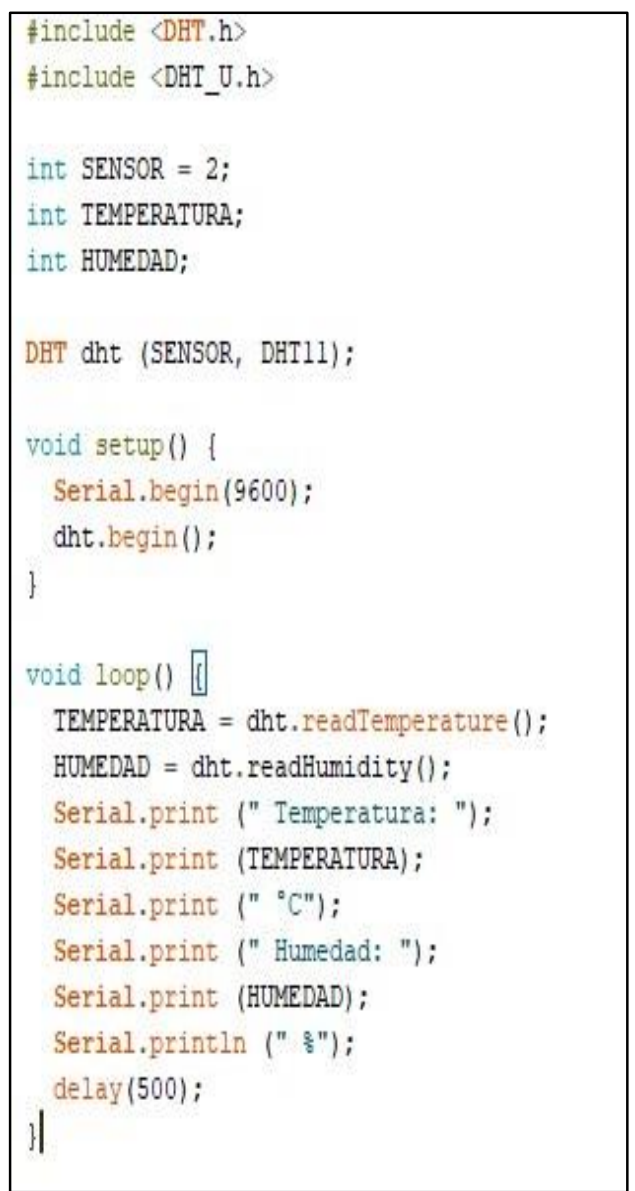

Figura 6 Código de programación sensor DHT11 Fuente: Elaboración Propia

De forma sencilla el código puede ser interpretado de la siguiente manera; se comienza añadiendo librerías especializadas para la lectura y deducción de las señales digitales del DHT11. Después se declaran las variables enteras. La variable "SENSOR" se asignará al pin número 2 para recibir las señales digitales del sensor. La variable "TEMPERATURA" representará en el módulo serial la señal de temperatura en ${ }^{\circ} \mathrm{C}$ y la variable "HUMEDAD" representará en el módulo serial la humedad relativa en valores de porcentaje 0 a $100 \%$.

\section{Construcción de la sección de medición de gas metano}

La siguiente sección en ser integrada al prototipo físico fue la que realiza la función de medir la variable de porcentaje de gas metano, una parte fundamental en el proyecto. De acuerdo al fundamento teórico, el sensor a implementarse fue el MQ-4, capaz de medir concentraciones de metano de 300 a 10000 ppm. (Rodríguez, Delgado, Aroca Trujillo, \& Navarrete Ramos, 2018)
Fue seleccionada tubería PVC hidráulico de $1 \frac{1}{2}$ " y el sensor digital DHT11 para medición de la humedad y temperatura. El elemento diseñado para ser implementado en el sistema y contener al sensor MQ-4, fue una abrazadera conformada por tres piezas, de las cuales, dos se unen físicamente por medio de tornillos con su respectiva tuerca y la tercera está diseñada para sujetar el sensor dentro de la abrazadera. Poseen igualmente desde una perspectiva radial una abertura central donde es acoplada la tubería de $1 \frac{1}{2}$ " y de manera axial en el centro, una de ellas posee un espaciamiento en la forma específica del sensor, con una guía por la que pueden ser guiados los cables que alimentan y comunican al sensor con la tarjeta.

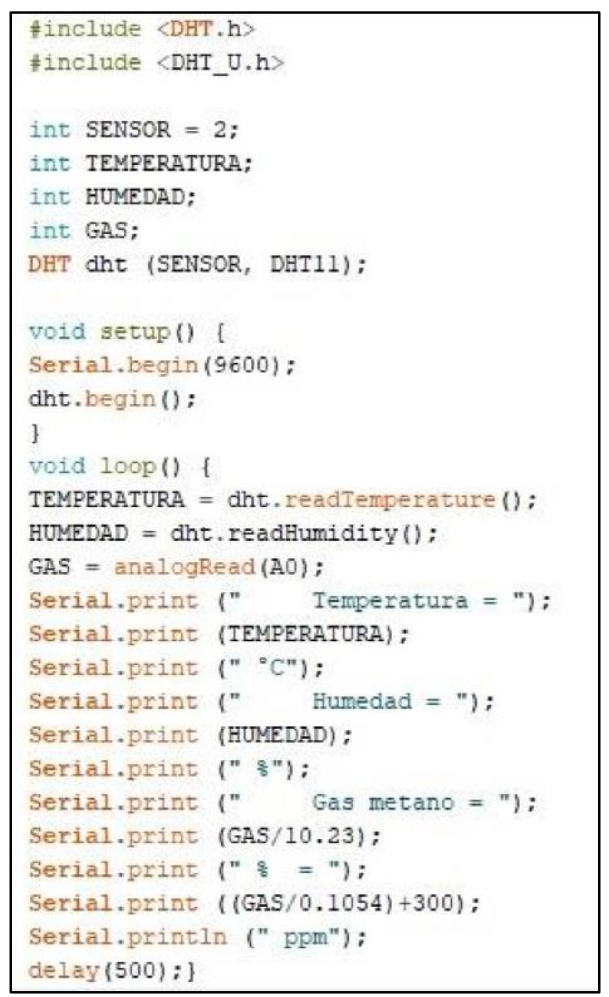

Figura 7 Código de programación sensor MQ-4 Fuente: Elaboración Propia

El código es interpretado de la siguiente manera; se comienza añadiendo librerías especializadas para la lectura y deducción de las señales digitales del DHT11. Después se declaran las variables enteras. $\mathrm{La}$ variable "SENSOR" se asignará al pin número 2 para recibir las señales digitales del sensor. La variable "TEMPERATURA" representará en el módulo serial la señal de temperatura en ${ }^{\circ} \mathrm{C}$ y la variable "HUMEDAD" representará en el módulo serial la humedad relativa en valores de porcentaje 0 a $100 \%$. La variable "GAS" representará la señal analógica enviada por el sensor de metano. 


\section{Construcción de la sección de desulfuración y deshumidificación}

Posteriormente se procedió a realizar la construcción de la sección de desulfuración y deshumidificación del gas. Para el prototipo físico fue seleccionado el método de adsorción por medio de gel de sílice $\left(\mathrm{SiO}_{2} \mathrm{H}_{2} \mathrm{O}\right)$. Usando dos coples de $1 \frac{1}{2} ", 15 \mathrm{~cm}$ de tubo PVC hidráulico de $1 \frac{1 / 2}{2}$ " y malla de mosquitero para permitir el fuljo del gas, se realizó un filtro que en su interior se le agregó 38 gramos de gel de sílice.

\section{Construcción del inversor monofásico}

Se realizó la construcción de un inversor monofásico de entrada a $12 \mathrm{~V} \mathrm{CD}$ a $127 \mathrm{~V} \mathrm{AC}$. El dispositivo se conectó a una batería de alimentación de 12VCD y mediante su configuración, el inversor dará como salida $127 \mathrm{~V}$ CA con una frecuencia aproximada de $60 \mathrm{~Hz}$.

Además, se empleó un transistor de potencia que es muy utilizado en electrónica de potencia el cual es el MOSFET. Este es un dispositivo semiconductor para la conmutación y amplificación de señales, su nombre completo es Transistor de Efecto de Campo de Metal Oxido Semiconductor, y esto se debe a la construcción del propio transistor. Para este proyecto se utilizó el MOSFET IRF44N, el cual debido a su configuración es requerido para el tipo de inversor.

Los pulsos PWM son gracias al circuito integrado CMOS CD4047 que su función es dar una salida de frecuencia de la cual podemos manipular mediante un potenciómetro que está conectado en el control de tiempo del multivibrador. Con esto será posible poder manejar la frecuencia necesaria para realizar la conmutación de los MOSFET.

\section{Resultados}

\section{Sensor DHT11}

Fue cargado el programa que se presentó en la sección de "Construcción de la sección de medición de temperatura y humedad" y arrojó los siguientes datos.

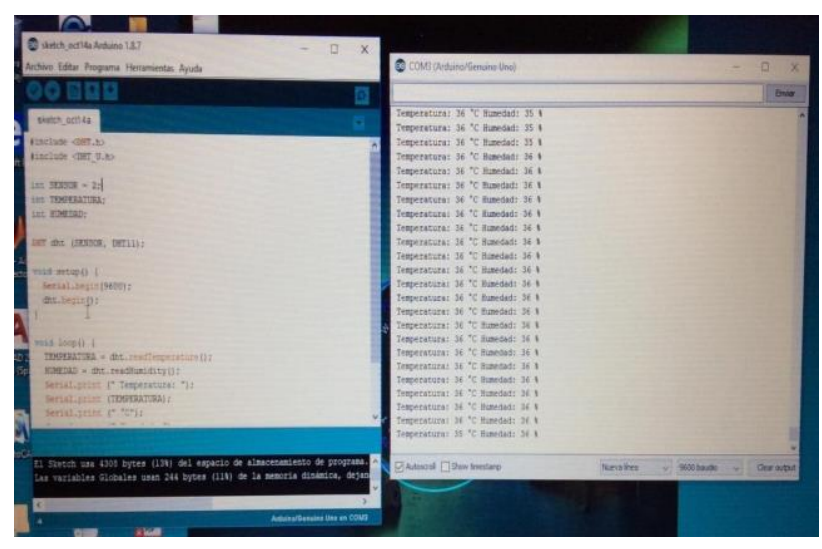

Figura 9 Resultados sensor DHT11

Fuente: Elaboración Propia

Durante las primeras pruebas en una zona ambiente daba valores aproximados a $30^{\circ} \mathrm{C}$ y $50 \%$ de humedad relativa. Al inyectarle aire caliente arrojó los datos de $36^{\circ} \mathrm{C}$ y una humedad relativa del $30 \%$ a $36 \%$, como se muestra en la imagen.

\section{Sensor MQ-4}

De manera semejante a la pieza del sensor DHT11, la abrazadera del sensor MQ4 fue ensamblada, conectada el sensor con su respectivo cableado hacia el microcontrolador y la sección fue integrada al sistema completo. Fue cargado el programa que se presentó en la sección de "Construcción de la sección de medición de gas metano" y arrojó los siguientes valores.

Al realizar las pruebas, no fue conectado el sensor DHT11 sino solo el MQ-4. En medida del porcentaje de metano en el ambiente comenzó arrojando valores del $3 \%$ un aproximado a $350 \mathrm{ppm}$ debido a que también este es poco sensible al dióxido de carbono (véase gráfica en el fundamento teórico) y otros gases combustibles. Para probar su eficacia, se realizaron pruebas usando gas butano de un encendedor, gas al cual también es sensible en una medida menor al metano. El monitor serial comenzó a arrojar resultados que llegaron hasta el $70 \%$ es decir 7800 ppm, cuando fue quitado el encendedor, los valores fueron reestableciéndose hasta llegar a los valores iniciales.

\section{Prototipo físico desarrollado}

Se realizó la unión de cada una de las secciones construidas para así obtener un prototipo físico. 
En la siguiente imagen se muestra primeramente como fuente de alimentación el circuito inversor monofásico que energiza los extractores. Después de los extractores se encuentran las reducciones, manguera y válvula de bola. De forma sucesiva se encuentra la abrazadera del sensor DHT11, la sección de deshumidificación representada por el filtro de gel de sílice, la abrazadera del sensor MQ-4 y la sección de desulfuración representada por el filtro de hidróxido de hierro

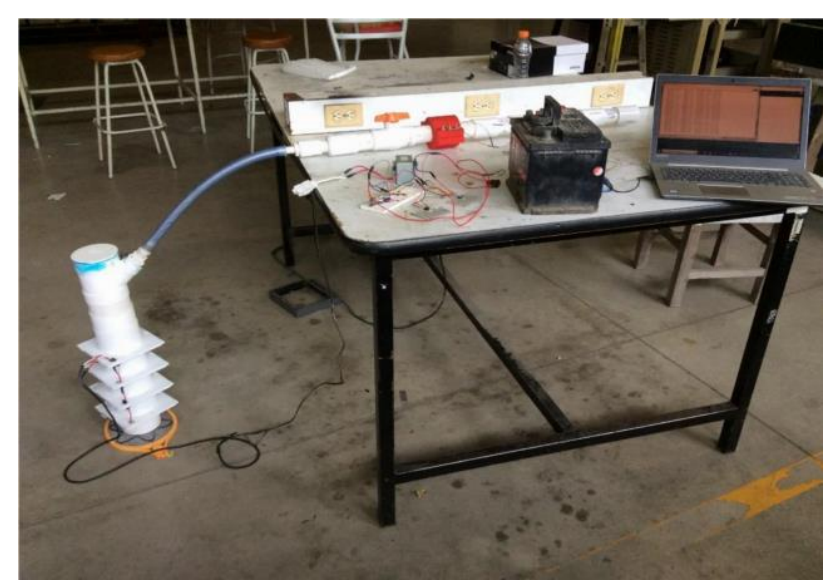

Figura 10 Prototipo físico vista 1

Fuente: Elaboración Propia

\section{Propuesta final del prototipo en diseño 3D}

Tomando como base las innovaciones hechas durante el desarrollo del proyecto, la primera propuesta de diseño en 2D, se procedió a realizar la propuesta final de diseño en 3D.

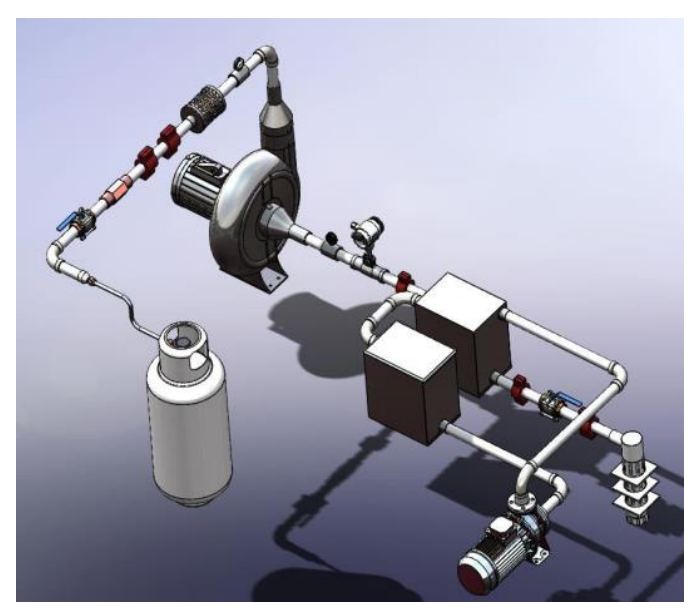

\section{Conclusiones}

Proveyendo una solución a la problemática, se realizó la construcción de un sistema prototipo portátil de extracción y tratamiento de gas metano. Siendo fundamentado en investigaciones de la rama físico-matemáticas como lo es la química, electricidad, electrónica e instrumentación.
Estas disciplinas nos brindaron una base para la elección de los procesos necesarios para la extracción y tratamiento del metano, elementos, materiales y dispositivos que realizaron esta tarea. Los procesos realizados en cada sección fueron la extracción inicial, separación de sólidos, deshumidificación, desulfuración y medición de las variables de porcentaje de gas metano, temperatura $y$ humedad.

Se proporcionó una alternativa novedosa la cual abona innovación y desarrollo tecnológico al Instituto Tecnológico José Mario Molina Pasquel Y Henríquez, sumando al plan estatal de desarrollo con un modelo de utilidad, que pueda ser presentado e incorporado a rellenos sanitarios en operación o que ya han sido cerrados y están actualmente incorporando gas metano a la atmosfera.

El prototipo quedó funcionando y a disposición del Instituto para trabajos futuros, con el fin de conseguir mejoras en los aspectos estéticos y funcionales; tales como los métodos de control, mejoramiento de procesos, sistema de alimentación eléctrica, las formas de interacción usuario - máquina, etc.

El aprovechamiento del gas metano en vertederos como biocombustible y fuente de energía renovable es una industria en pleno auge en la actualidad, países alrededor del mundo, incluyendo México ya con algunas empresas en el mercado, están apostando a la energía limpia del metano. Reconociendo su valor como fuente de energía y ayuda al medio ambiente.

El creciente desperdicio y liberación de este gas de efecto invernadero puede ser reducida. Un sistema de extracción y tratamiento de gas metano provee una alternativa útil para este propósito y además el arranque de futuros proyectos a superior escala y mayor impacto, tanto en el área energética y ambiental como también en el área tecnológica y de innovación.

\section{Referencias}

Colmenares, W., \& Santos, K. (Mayo de 2007). Generación y manejo de gases en sitios de disposición final. Recuperado el Mayo de 2018, de Ingeniería química.

Coronado, S. (11 de Agosto de 2014). EL FINANCIERO. Obtenido de Impulsa 
Bioenergía de Nuevo León uso de biogás:

http://www.elfinanciero.com.mx/monterrey/imp ulsa-bioenergia-de-nuevoleon-uso-de-biogas

Garzón Pinto, N. (2017). Filogenia de los seres vivos: Dominio Achaea. Sevilla España: Universidad de Sevilla, Facultad de farmacia.

Llamas, L. (16 de Octubre de 2016). Detector de gases con Arduino y la familia de sensores $M Q$. Obtenido de Luis Llamas. Ingeniería, Informática y Diseño: https://www.luisllamas.es/arduino-detector-gas$\mathrm{mq} /$

López Arriaza, D. Á., \& Núñez Lazo, L. (2016). Universidad de Chile. Recuperado el Abril de 2018, de Facultad de Ciencias Físicas y Matemáticas, Departamento de Ingeniería Mecánica:

http://repositorio.uchile.cl/bitstream/handle/225 0/138545/Modelo-para-eldiseno-de-sistemasde-captacion-y-aprovechamiento-debiogasproducido.pdf? sequence $=1$

Luque, M. (s.f.). La fuente olvidada de metano. Obtenido de Solo Ciencia: https://www.solociencia.com/quimica/0602137 9.htm

Mapas, G. (s.f.). Mapa del Relleno Sanitario El Gavilán. Obtenido de Puerto Vallarta Jalisco: http://satellites.pro/Mapa_del_Mundo\#20.7621

48,-105.137654,18

MECHILE, G. d., PNUD, FAO, \& GEF. (2011). Manual de Biogás. Santiago de Chile.

Muhammad H, R. (1993). Electrónica de potencia, Circuitos, dispositivos y aplicaciones. Fort Wayne, Indiana: Prentice Hall.

NORMA, O. M. (2004). NOM-083SEMARNAT-2003 Especificaciones de protección ambiental para la selección del sitio, diseño, construcción, operación, monitoreo, clausura y obras complementarias de un sitio de disposición final de residuos sólidos urbanos y de manejo especial. México Distrito Federal: DIARIO OFICIAL DE LA FEDERACIÓN.
Noticias Ciencia, F. (s.f.). Obtenido de http:/www.laflecha.net/canales/ciencia/noticias/ una-bacteria-que-sealimenta-de-metano-podríaservir-para-combatir-el-calentamiento-global NOTIMEX. (8 de Agosto de 2013). NL transforma en energía 4,500 toneladas diarias de basura. Obtenido de ULMA: http://obrasweb.mx/construccion/2013/08/08/nl -transforma-en-energia-4500toneladas-diariasde-basura

Ordoñez Mendoza, A., Fritz Rodríguez, E., \& Espinosa Solares, T. (2017). Determinación de concentración de metano con sensores semiconductores. Texcoco de Mora, México: ECORFAN.

Ortega, V., Rodríguez, S., Fernández, E., \& Bárcenas, L. (Abril de 2015). Ingeniería Hidráulica y Ambiental. Recuperado el Mayo de 2018, de Principales métodos para la desulfuración del biogás: http://scielo.sld.cu/scielo.php?pid=S168003382015000100004\&script $=$ sci_arttext\&tlng= $\mathrm{pt}$

Pichardo, V. (2016). Metano, fuente alternativa de energía. Capital México.

Quezada Barrón, M. (10 de Julio de 2009). Aprueban a Biogás de Juárez producir energía a través del gas metano. Ciudad Juárez, Chihuahua, México.

Rodríguez, R., Delgado, D., Aroca Trujillo, J., \& Navarrete Ramos, A. (2018). Diseño $e$ implementación de un sistema de monitoreo de emisión de gases en el cultivo del arroz. Neiva, Colombia: Corporación Universitaria del Huila.

Rubio Serrano, F. (2019). IMASA, Ingeniería y proyectos S.A. Obtenido de Ciclo higroscópico: http://www.ciclohigroscopico.com/compuestoshigroscopicos/

Sara, L. (12 de Noviembre de 2015). Técnicas de deshumidificación. Obtenido de Climatización10.

Tinajero Mendoza, R. (2017). Estimación del biogás generado en el relleno sanitario El Gavilán de Puerto Vallarta. Puerto Vallarta Jalisco: Instituto Tecnológico Superior de Puerto Vallarta. 
Vaquerano Pineda, N., Salazar Rojas, T., \& Porras Acosta, M. (2016). Medición automática del metano en biogás por columnas de desplazamiento. Cartago, Costa Rica: Instituto Tecnológico de Costa Rica.

Vélez Pereira, A., \& Camargo Caicedo, Y. (Septiembre de 2009). ResearchGate. Obtenido de Emisiones de Biogás producidas en rellenos sanitarios:

https://www.researchgate.net/publication/27606 2721 Wade, L. J. (2012). Química orgánica. México: PEARSON. 